\title{
Heteroepitaxial Growth of Ge Nanowires on Si Substrates
}

\author{
Pietro Artoni, ${ }^{1,2}$ Alessia Irrera, ${ }^{1}$ Emanuele Francesco Pecora, ${ }^{1,2}$ \\ Simona Boninelli, ${ }^{1}$ Corrado Spinella, ${ }^{3}$ and Francesco Priolo ${ }^{1,2}$
}

${ }^{1}$ MATIS IMM CNR, Via S. Sofia 64, 95123 Catania, Italy

${ }^{2}$ Dipartimento di Fisica e Astronomia, Università di Catania, Via S. Sofia 64, 95123 Catania, Italy

${ }^{3}$ IMM CNR, VIII Strada 5, 95121 Catania, Italy

Correspondence should be addressed to Alessia Irrera, alessia.irrera@ct.infn.it

Received 26 September 2011; Revised 11 November 2011; Accepted 14 November 2011

Academic Editor: Fabrice Gourbilleau

Copyright () 2012 Pietro Artoni et al. This is an open access article distributed under the Creative Commons Attribution License, which permits unrestricted use, distribution, and reproduction in any medium, provided the original work is properly cited.

\begin{abstract}
Electron beam evaporation has been used to prepare Ge nanowires (NWs) on top of (111) Si substrates. Despite the nonUHV growth conditions, scanning and transmission electron microscopies demonstrate that NWs are single crystal with specific crystallographic growth directions ([111], [110], and [112]). NWs are faceted, exhibiting the lower energy plans on the surface. The faceting depends on the growth direction. Moreover, the detrimental effects for Ge NWs growth of O atoms contamination are discussed. Finally, we describe how a proper preparation of the Au catalyst is able to increase the Ge NW density by a factor of 4 , while heteroepitaxy and faceting features are maintained.
\end{abstract}

\section{Introduction}

Semiconductor nanowires (NWs) are a new class of quasione-dimensional materials. These structures show novel physical properties, such as electron band-gap modulation through NW size control [1], ballistic transport regime at room temperature $[2,3]$, and interesting chemical properties, due to their low dimensionality and to their high surface-volume ratio. These properties make these objects very promising building blocks for nanocircuits [4], sensors [5], nanophotonic devices [6], and solar cells [7]. Moreover, Ge has recently stimulated interest in the scientific community, because it may overcome some limits of conventional CMOS technology, which is based primarily on Si. In fact, Ge has higher electron and hole mobility than Si. The smaller bandgap of Ge may be desirable for indoor solar cells [7] and detectors [8], in which absorption at longer wavelengths is required. Moreover, exciton Bohr radius in Ge $(24.3 \mathrm{~nm})$ is about five times larger than in $\mathrm{Si}(4.8 \mathrm{~nm})$, and this property makes Ge confined structures for carriers easier to be realized than those based on Si.

Ge NWs are typically synthesized through a self-assembling method, the Vapor-Liquid-Solid mechanism [9] using chemical vapor deposition techniques (CVD). Orientation and surface faceting of Ge NWs prepared by CVD have been studied $[10,11]$. These are key features for the control of Ge NW optical and electrical properties. On the other side, physical vapor deposition (PVD) techniques allow a more accurate control of the parameters of growth; at the same time, they allow the study of growth mechanisms and definitively permit to control structural properties of these nanostructures. Concerning this, electron beam evaporation (EBE) gives, unlike other PVD techniques, the opportunity of evaporating under non-UHV conditions, therefore representing a low-cost technique ideal for a CMOS process integration. It has been recently demonstrated that EBE is a good method to obtain heteroepitaxial growth of faceted Ge NWs on (111)-oriented Si substrate despite the non-UHV conditions $[12,13]$.

Atoms diffusion during the first stages of the growth process is strategic to definitely obtain NWs in both physical and plasma-enhanced chemical vapor deposition techniques $[14,15]$. Concerning this, a crucial parameter which has a tremendous effect on diffusion is oxygen presence $[16,17]$. Therefore, the study of the influence of oxygen contaminations on Ge NWs growth is fundamental because it is well 


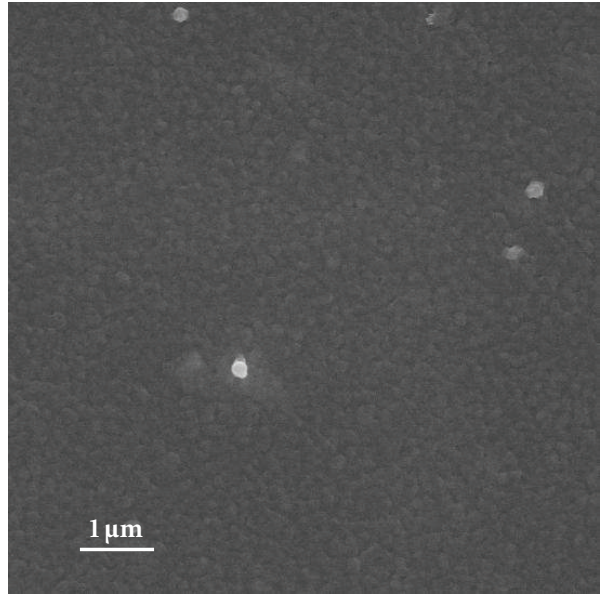

(a)

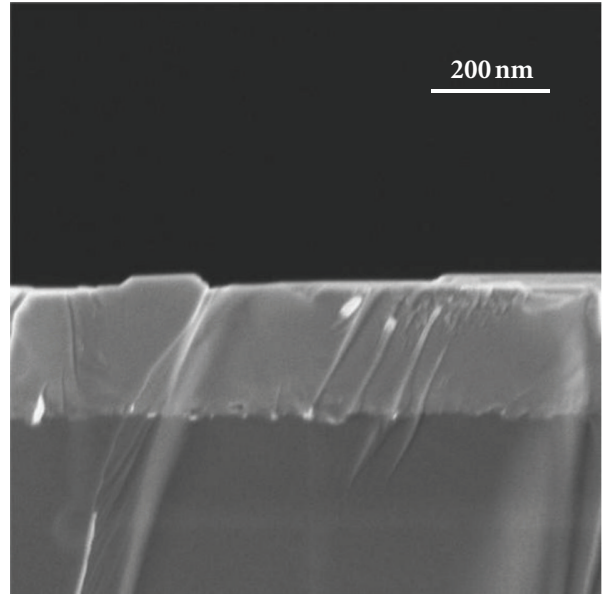

(b)

FIgURE 1: (a) Top view SEM image of an Si substrate covered with Au evaporated at room temperature and Ge evaporated at $480^{\circ} \mathrm{C}$; native $\mathrm{SiO}_{2}$ has not been removed before Ge evaporation. (b) Cross-section SEM image of the sample shown in (a). The 2D Ge layer on Si substrate is clearly visible.

known that Ge surface diffusion strongly depends on the substrate characteristics $[14,18]$.

In this paper we investigate the influence of $\mathrm{O}$ atom contaminations on the early stages of the heteroepitaxial growth of Ge NWs on Si substrates and the impact of the Au catalyst preparation method on the NW density.

\section{Experimental}

Ge NWs were grown on n-type (111)-oriented Si substrates. UV oxidation and HF etching were performed to remove any surface contamination and the native oxide layer before loading the samples into the evaporation chamber (base pressure: $1-2 \times 10^{8} \mathrm{mbar}$ ), where both $\mathrm{Au}$ and $\mathrm{Ge}$ were evaporated by focusing an electron beam on high-purity $\mathrm{Au}$ pellets or Ge ingots. The deposition rate and film thickness were controlled in situ by a quartz microbalance and checked ex situ by Rutherford backscattering spectrometry. The current of the electron gun was set to ensure a flux of $1.5 \times$ $10^{14}$ atoms $/ \mathrm{cm}^{2} \mathrm{~s}$ for all the samples. During the deposition, the substrate was eventually resistively heated. Two different synthesis procedures were employed. In the first case, a $2 \mathrm{~nm}$-thick Au layer was evaporated at room temperature (RT). After Au deposition, samples were annealed at $700^{\circ} \mathrm{C}$ for $2 \mathrm{~h}$ to induce the continuous layer breaking and the formation of $\mathrm{Au}$ clusters on the $\mathrm{Si}$ substrate. Then, the temperature was set to $480^{\circ} \mathrm{C}$ for $\mathrm{Ge}$ evaporation. In the second case, the same $\mathrm{Au}$ fluence was evaporated at the same Ge NWs growth temperature of $480^{\circ} \mathrm{C}$, and $\mathrm{Ge}$ was subsequently evaporated without cooling the sample. The evaporated Ge fluence was fixed to $0.8 \times 10^{18}$ atoms $/ \mathrm{cm}^{2}$ for all the samples. Structural characterization was performed by a Field-Emission Scanning Electron Microscope (FESEM, Zeiss Supra 25) and a $200 \mathrm{kV}$ Transmission Electron Microscope (TEM, JEOL JEM 2010). NW statistical analysis has been conducted by Gatan digital microscope software.
The lattice simulations have been performed by CaRIne crystallography software.

\section{Results and Discussions}

3.1. Influence of $O$ Contamination. To clarify the effect of $O$ contamination on Ge NWs growth, we prepared two Si samples with different surface pretreatments. In particular both samples were HF etched before Au deposition, performed at RT. After the thermal annealing, necessary for the formation of the Au clusters, one of the two samples was exposed to air till the formation of the native oxide layer, while the other was maintained in vacuum. Finally, the same Ge fluence was evaporated on both samples, one being oxygen-free and the other with $\mathrm{O}$ contaminations between the Au clusters. A topview SEM image of the O-contaminated sample is reported in Figure 1(a), in which a negligible density of gold clusters is visible, compared to their initial density (about $10^{8} \mathrm{~cm}^{-2}$ ). This density drastically decreases due to the formation of a 2D Ge layer on Si substrate which buries most of these gold clusters. The cross-section SEM view of the 2D Ge overgrown layer is shown in Figure 1(b). There is no NWs growth. Moreover, 2D layers formation typically occurs in physical vapor deposition techniques, such as EBE [14], MBE [19], or in UHV CVD technique [20]. On the other hand, Figure 2 shows a typical low-magnification SEM image of the oxygenfree sample. In this case the NWs are clearly present and the sample surface exhibits the triangular terraces typical of an epitaxial 2D growth along (111) planes.

In PVD growth, the diffusion of ad-atoms on the substrate plays a fundamental role $[15,21]$. We already demonstrated [12] that two independent processes contribute to Ge NWs growth by EBE: direct impingement of $\mathrm{Ge}$ atoms on the catalyst droplet and diffusion of $\mathrm{Ge}$ ad-atoms from the surface along the NW sidewalls up to the top of it. Ge atoms coming from vapor phase, which 


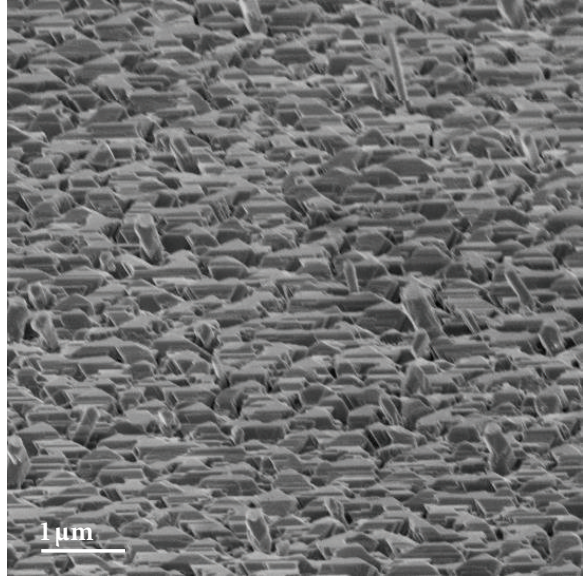

FIGURE 2: $65^{\circ}$ tilt view SEM image of a Si substrate covered with Au evaporated at room temperature and $\mathrm{Ge}$ evaporated at $480^{\circ} \mathrm{C}$. The sample is oxygen-free.

cannot diffuse to the NW, contribute to form a $2 \mathrm{D} \mathrm{Ge}$ layer on Si substrate. Ge NWs are obtained through the competition between axial growth of the NWs versus 2D Ge layer growth. The ad-atoms contribution is necessary to make the NW growth faster than that of the $2 \mathrm{D} G \mathrm{Ge}$ layer. It is well known that $\mathrm{Ge}$ surface diffusivity on $\mathrm{SiO}_{2}$ is several orders of magnitude lower than that on Si or Ge. So, the presence of $\mathrm{O}$ contaminations strongly reduces the ad-atoms mobility on the surface between Au droplets inhibiting the NW growth. This is a further demonstration of the fundamental role played by diffusion in Ge NW growth.

3.2. Crystallographic Properties. The sample shown in Figure 2 was prepared according to the first synthesis described in Section 2 , that is, the Au was evaporated at RT and a subsequent thermal annealing was conducted in order to form the clusters. Ge NWs grown according to this procedure exhibit specific tilt angles with respect to the (111) Si substrate. The observed NW density is about of $5 \times$ $10^{6} \mathrm{~cm}^{-2}$ and the measured NW mean radius is $100 \pm 40 \mathrm{~nm}$. We analyzed a large amount of SEM images and, according to the substrate orientation, we simulated all the possible heteroepitaxial growth directions. Then, we compared the simulations with the experimental images and determined that NWs have only three specific crystallographic directions belonging to the $[111,110]$ or $[112]$ family. We never observed NWs forming random angles with the substrate or belonging to different growth directions. This is a clear evidence about the heteroepitaxial nature of Ge NWs on a Si substrate.

These results have been confirmed by TEM analyses, made on each family of NWs. For this purpose, Ge NWs were mechanically scratched from the substrate and put on a copper grid. Figure 3 shows a bright field TEM image of a [110] Ge NW. The inset shows the diffraction pattern obtained by a selected area of this NW. Different selected areas of the same NW show the same diffraction pattern.

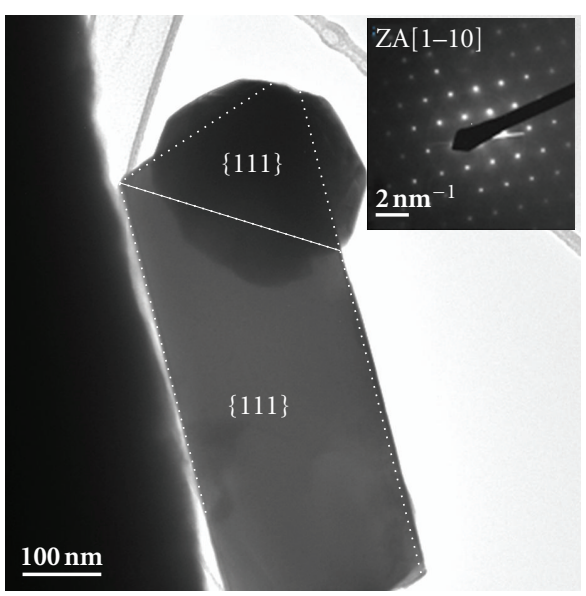

Figure 3: Bright-field TEM image of a [110]-oriented NW. The simulation of the corresponding faceted structure is superimposed. In the inset, the diffraction pattern of the NW corresponding to the [1-10] Zone Axis (ZA) is shown.

This result demonstrates that Ge NW structure is defect-free and monocrystalline. We compared the observed diffraction pattern with the one obtained in the [1-10] Zone Axis (ZA) by simulating the bulk lattice structure of Ge, superimposed on the TEM image. The experimental pattern is in perfect agreement with the theoretical one. Furthermore, each NW family exhibits a well-defined geometrical surface. We reconstructed the real lattice orientation and the crystallographic faces of [110]-oriented Ge NW, finding a rhomboidal shape based only on $\{111\}$ surface planes, with two $\{111\}$ planes on top. [110]-oriented nanowires with hexagonal shape, grown by gold-nanocrystal-seeded supercritical fluid-liquidsolid and containing four $\{111\}$ planes and two $\{100\}$ planes, have been observed [11], while in our works by EBE we observe for this specific orientation only $\{111\}$ planes. This could be due to a lateral overgrowth of the four $\{111\}$ planes on missing two $\{100\}$ planes, as recently observed in Ge NWs grown by MBE [22]. Part of the Ge adatoms of surface can be incorporated on the $\{111\}$ sidewalls, rather than contributing to axial growth of the NWs. We determined full accordance between the faceted shape of this NW and these crystallographic planes. This result can be related to the fact that $\{111\}$ planes are among the lowest energy plans of the diamond structure of Ge [23, 24] confirming that EBE conditions of growth are not too far from thermodynamic equilibrium. TEM image also shows the presence of the catalyzing Au droplet on one of the two upper faces. According to this, we suggest that, during the growth, the Au droplet could stay on one face only, acting as a sink for atoms which diffuse from NW sidewalls and for atoms directly impinging on it. These atoms ultimately contribute to a quasi-equilibrium growth of the Ge NW. Moreover, we observed faceting also on [112] NWs. These ones have a rectangular shape, with lateral faces oriented in opposite manner: two facets exhibit $\{111\}$ planes, the other ones exhibit $\{110\}$ planes. 


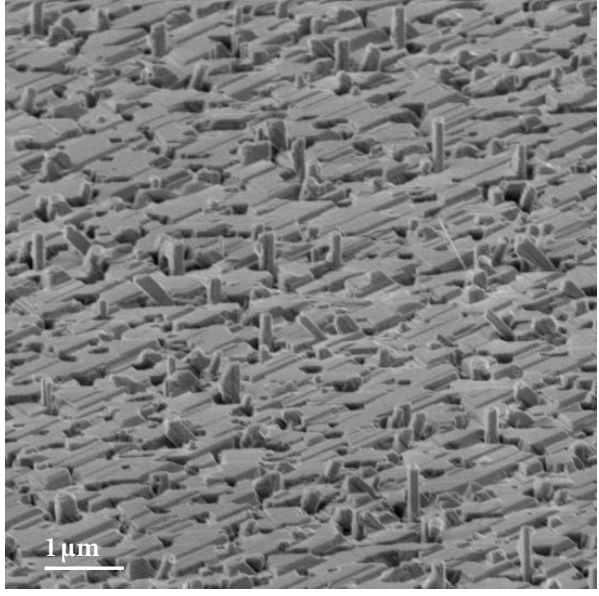

FIGURE 4: $65^{\circ}$ tilt view SEM image of a sample with both Au and Ge evaporated at $480^{\circ} \mathrm{C}$.

\subsection{Influence of Au Catalyst Preparation. A higher NW den-} sity would be desirable for applications. We prepared specific samples following a different catalyst preparation procedure in order to increase NWs density, as it has been demonstrated for Si NWs [16]. In particular, Au was evaporated at the same temperature of the NWs growth process, that is, $480^{\circ} \mathrm{C}$, and the sample was maintained heated during the switching between $\mathrm{Au}$ and $\mathrm{Ge}$ evaporation. Figure 4 shows a low-magnification SEM image of the sample prepared following this catalyst preparation procedure. We conducted a complete statistical analysis of the structural features of these NWs. Despite the different catalyst preparation, we are able to identify the same crystallographic families of the NWs growth direction. Thus, both heteroepitaxy and the relative percent of each crystallographic family are maintained and the same faceted shapes have been observed for [110] and [112] families of Ge NWs. Remarkably, this sample shows a density of Ge NWs equal to $2 \times 10^{7} \mathrm{~cm}^{-2}$, which is 4 times higher than the one obtained by evaporating Au at RT.

It is noteworthy that the Au droplet densities obtained with the two different methods of catalyst preparation are comparable, as reported in plan-view SEM analyses of Figure 5. In other words, despite the fact that the available Au cluster and the NW growth conditions are the same, we obtain a different effective NWs density. This means that the active Au droplet concentration changes by modifying the catalyst synthesis. In order to explain this evidence, it should be noted that the competition between the axial and the planar growth rate is a key issue in the EBE growth mechanism. In fact, the droplet has to become active (i.e., the eutectic conditions are reached and the axial rate overcomes the planar one) before it is covered by the $2 \mathrm{D}$ layer. By evaporating $\mathrm{Au}$ on the heated substrate, Au atoms have sufficient mobility to form clusters, which, in turn, have immediately the possibility to form the liquid eutectic with the Si substrate becoming active for promoting the NW growth. In fact, by evaporating Ge atoms, they diffuse into the Au liquid droplet, definitely keeping the droplet

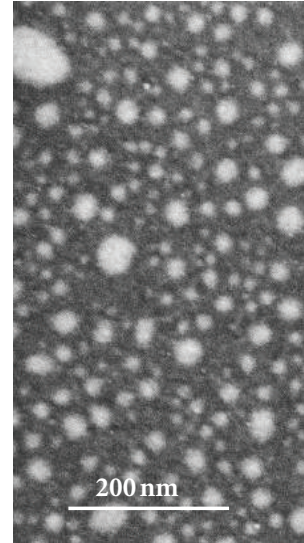

(a)

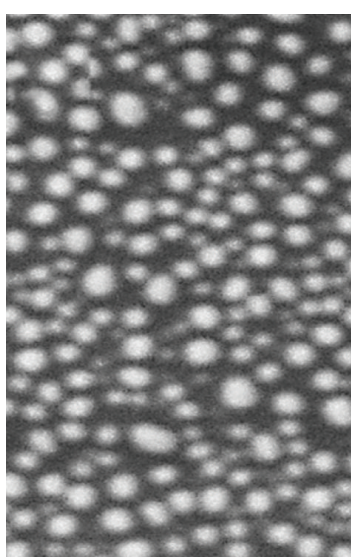

(b)
Figure 5: (a) Plan-view SEM image of a sample deposited with $2 \mathrm{~nm}$-thick Au layer and sequent annealed at $700^{\circ} \mathrm{C}$ for two hours. (b) Plan-view SEM image of a sample in which the same gold thickness has been evaporated directly at the same NWs growth temperature of $480^{\circ} \mathrm{C}$.

always liquid anyway, until the eutectic conditions $\mathrm{Au} / \mathrm{Ge}$ are reached and Ge NW growth can start.

On the other hand, in the catalyst preparation procedure involving Au evaporation at RT, we first break the continuous Au layer to form the clusters. Then the sample is cooled at RT and as a consequence Au clusters solidify. Afterward the sample is heated again at the growth temperature and Ge is evaporated. Au clusters can form the liquid eutectic just now, and some time is required before it becomes active for the NW growth. During this time, the 2D layer growth covers some of the clusters, and thereof the NW density decreases.

The Ge evaporation process is the same for both of the two samples with different catalyst preparations. This means that Ge ad-atoms kinetic processes during Ge NWs growth are the same independently from the way of preparation of the catalyst. In fact we did not observe any variation in NW lengths.

\section{Conclusions}

Heteroepitaxial growth of single-crystal Ge NWs on (111)oriented Si wafer via EBE technique has been demonstrated through SEM and TEM analyses. We found that specific surface crystallographic planes ( $\{111\}$ or $\{110\})$ correspond to each growth direction. We have proved that Ge surface diffusion is the key aspect of this growth. As a consequence, the presence of any $\mathrm{O}$ contamination reduces the ad-atoms diffusivity. In fact, we have demonstrated that the presence of $\mathrm{O}$ atoms is detrimental for Ge NW growth: a very thin $\mathrm{SiO}_{2}$ layer strongly influences both $\mathrm{Ge}$ and $\mathrm{Au}$ diffusion on the surface during the early stages of growth and definitely inhibits it. Furthermore, the high diffusivity of Ge allows the realization of faceted structures. At the same time, Ge surface diffusion, which is active since the early stages of growth, makes Ge feeding of Au droplets a very efficient process, both in the case of evaporating Au at RT with subsequent 
annealing and still more in the case of evaporating $\mathrm{Au}$ at the NW growth temperature. For this reason we observed an increase of the Ge NW density by a factor of 4 by changing the method of catalyst preparation.

\section{Acknowledgments}

The authors thank Fabio Iacona for useful discussions and Carmelo Percolla and Corrado Bongiorno for the expert technical assistance.

\section{References}

[1] M. S. Gudiksen, J. Wang, and C. M. Lieber, "Size-dependent photoluminescence from single indium phosphide nanowires," Journal of Physical Chemistry B, vol. 106, no. 16, pp. 4036-4039, 2002.

[2] X. Zhou, S. A. Dayeh, D. Aplin, D. Wang, and E. T. Yu, "Direct observation of ballistic and drift carrier transport regimes in InAs nanowires," Applied Physics Letters, vol. 89, no. 5, Article ID 053113, 2006.

[3] W. Lu, J. Xiang, B. P. Timko, Y. Wu, and C. M. Lieber, "One-dimensional hole gas in germanium/silicon nanowire heterostructures," Proceedings of the National Academy of Sciences of the United States of America, vol. 102, no. 29, pp. 10046-10051, 2005.

[4] Y. Huang, X. Duan, Y. Cui, L. J. Lauhon, K. H. Kim, and C. M. Lieber, "Logic gates and computation from assembled nanowire building blocks," Science, vol. 294, no. 5545, pp. 1313-1317, 2001.

[5] Y. Cui, Q. Wei, H. Park, and C. M. Lieber, "Nanowire nanosensors for highly sensitive and selective detection of biological and chemical species," Science, vol. 293, no. 5533, pp. 1289-1292, 2001.

[6] M. H. Huang, S. Mao, H. Feick et al., "Room-temperature ultraviolet nanowire nanolasers," Science, vol. 292, no. 5523, pp. 1897-1899, 2001.

[7] E. Garnett and P. Yang, "Light trapping in silicon nanowire solar cells," Nano Letters, vol. 10, no. 3, pp. 1082-1087, 2010.

[8] Y. H. Ahn and J. Park, "Efficient visible light detection using individual germanium nanowire field effect transistors," Applied Physics Letters, vol. 91, no. 16, Article ID 162102, 2007.

[9] R. S. Wagner and W. C. Ellis, "Vapor-liquid-solid mechanism of single crystal growth," Applied Physics Letters, vol. 4, no. 5, pp. 89-90, 1964.

[10] H. Adhikari, A. F. Marshall, C. E. D. Chidsey, and P. C. McIntyre, "Germanium nanowire epitaxy: shape and orientation control," Nano Letters, vol. 6, no. 2, pp. 318-323, 2006.

[11] T. Hanrath and B. A. Korgel, "Crystallography and surface faceting of germanium nanowires," Small, vol. 1, no. 7, pp. 717-721, 2005.

[12] E. F. Pecora, A. Irrera, P. Artoni et al., "Heteroepitaxial growth and faceting of Ge nanowires on $\mathrm{Si}(111)$ by electron-beam evaporation," Electrochemical and Solid-State Letters, vol. 13, no. 5, pp. K53-K55, 2010.

[13] A. Irrera, E. F. Pecora, and F. Priolo, "Control of growth mechanisms and orientation in epitaxial Si nanowires grown by electron beam evaporation," Nanotechnology, vol. 20, no. 13, Article ID 135601, 2009.

[14] P. Artoni, E. F. Pecora, A. Irrera, and F. Priolo, "Kinetics of si and ge nanowires growth through electron beam evaporation," Nanoscale Research Letters, vol. 6, no. 1, article 162, pp. 1-8, 2011.

[15] V. G. Dubrovskii, N. V. Sibirev, J. C. Harmand, and F. Glas, "Growth kinetics and crystal structure of semiconductor nanowires," Physical Review B, vol. 78, no. 23, Article ID 235301, 2008.

[16] E. F. Pecora, A. Irrera, and F. Priolo, "Influence of O contamination and Au cluster properties on the structural features of Si nanowires," Thin Solid Films, vol. 518, no. 9, pp. 2562-2564, 2010.

[17] S. Kodambaka, J. B. Hannon, R. M. Tromp, and F. M. Ross, "Control of Si nanowire growth by oxygen," Nano Letters, vol. 6, no. 6, pp. 1292-1296, 2006.

[18] V. Cherepanov and B. Voigtländer, "Influence of material, surface reconstruction, and strain on diffusion at the $\mathrm{Ge}(111)$ surface," Physical Review B, vol. 69, no. 12, Article ID 125331, pp. 1253311-1253318, 2004.

[19] L. Schubert, P. Werner, N. D. Zakharov et al., "Silicon nanowhiskers grown on (111) Si substrates by molecularbeam epitaxy," Applied Physics Letters, vol. 84, no. 24, pp. 4968-4970, 2004.

[20] J. W. Dailey, J. Taraci, I. Clement, D. J. Smith, J. Drucker, and S. T. Picraux, "Vapor-liquid-solid growth of germanium nanostructures on silicon," Journal of Applied Physics, vol. 96, no. 12, article 12, pp. 7556-7567, 2004.

[21] J. C. Harmand, M. Tchernycheva, G. Patriarche, L. Travers, F. Glas, and G. Cirlin, "GaAs nanowires formed by Au-assisted molecular beam epitaxy: effect of growth temperature," Journal of Crystal Growth, vol. 301-302, pp. 853-856, 2007.

[22] T. Xu, J. Sulerzycki, J. P. Nys, G. Patriarche, B. Grandidier, and D. Stiévenard, "Synthesis of long group IV semiconductor nanowires by molecular beam epitaxy," Nanoscale Research Letters, vol. 6, no. 1, article 113, pp. 1-7, 2011.

[23] J. M. Bermond, J. J. Métois, X. Egéa, and F. Floret, "The equilibrium shape of silicon," Surface Science, vol. 330, no. 1, pp. 48-60, 1995.

[24] J. M. Zhang, F. Ma, K. W. Xu, and X. T. Xin, "Anisotropy analysis of the surface energy of diamond cubic crystals," Surface and Interface Analysis, vol. 35, no. 10, pp. 805-809, 2003. 


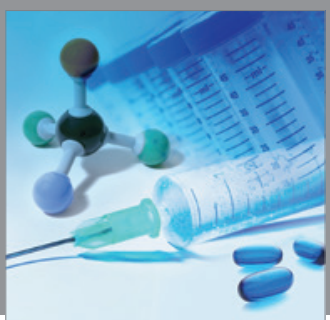

International Journal of

Medicinal Chemistry

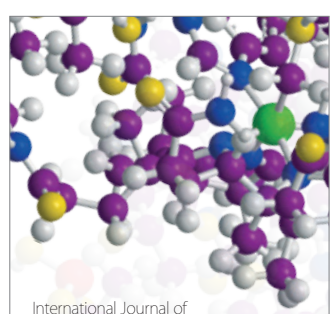

Carbohydrate Chemistry

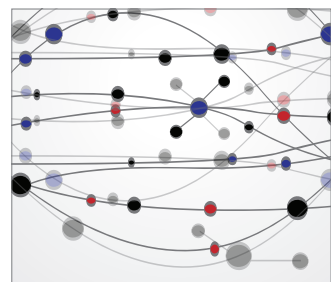

The Scientific World Journal
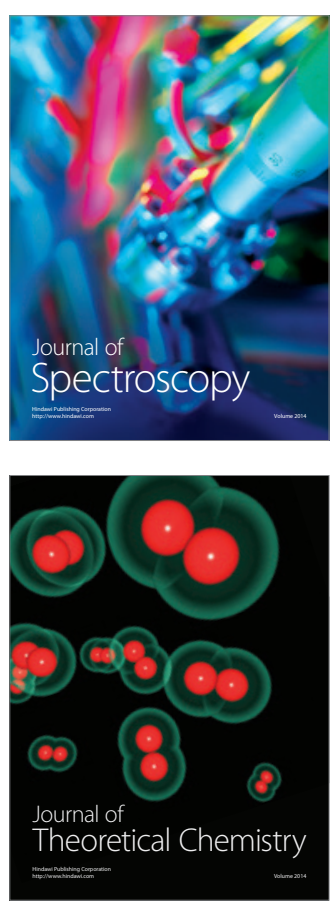
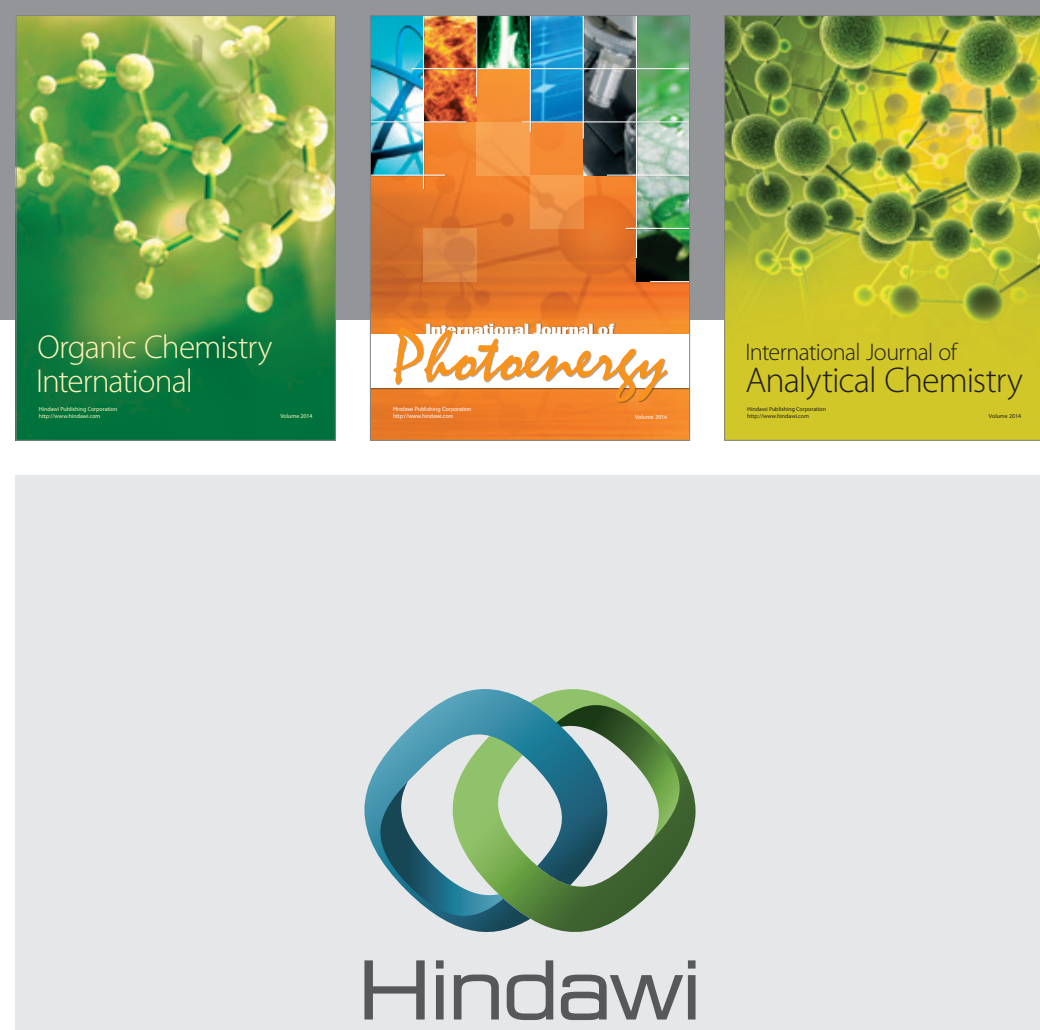

Submit your manuscripts at

http://www.hindawi.com
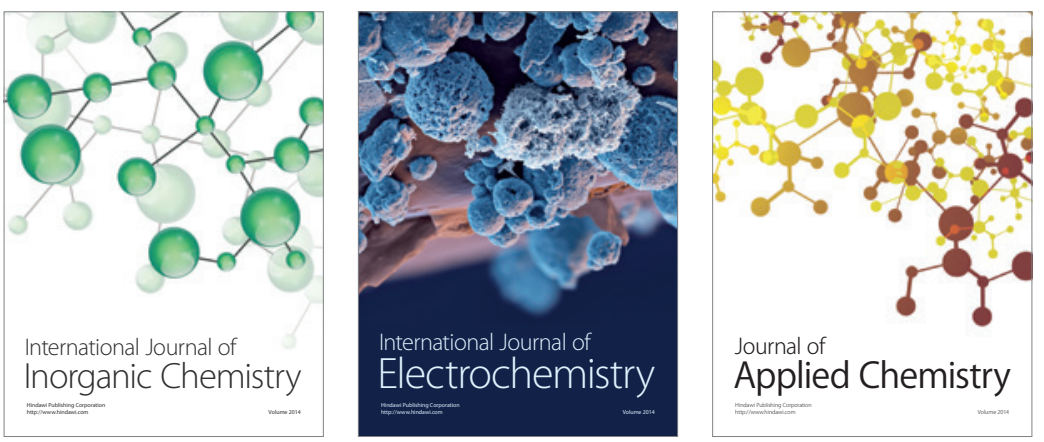

Journal of

Applied Chemistry
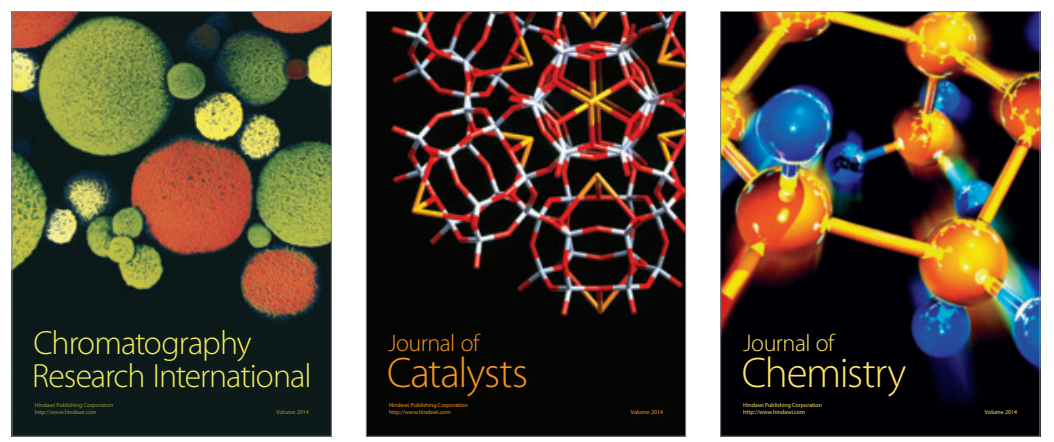
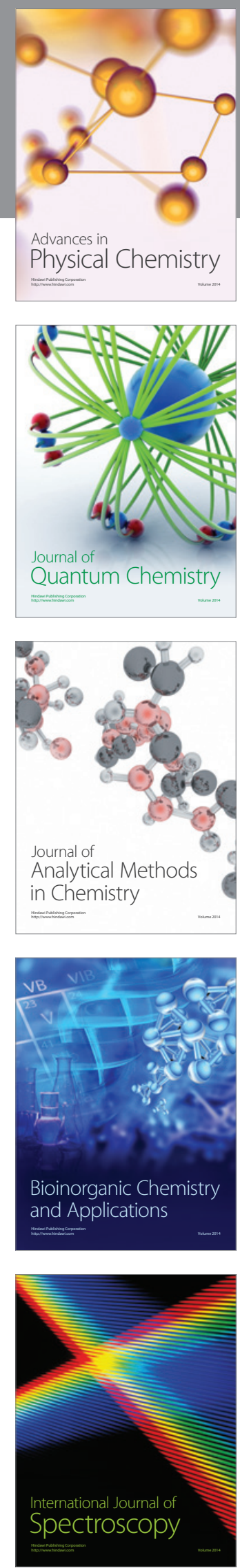